\title{
Performance of Binary Block Codes at Low Signal-to-Noise Ratios
}

\author{
Chi-Chao Chao, Member, IEEE, Robert J. McEliece, Fellow, IEEE, Laif Swanson, Member, IEEE, \\ and Eugene R. Rodemich
}

\begin{abstract}
The performance of general binary block codes on an unquantized additive white Gaussian noise (AWGN) channel at low signal-to-noise ratios is considered. Expressions are derived for both the block-error and the bit-error probabilities near the point where the bit signal-to-noise ratio is zero. These expressions depend on the global geometric structure of the code, although the minimum distance still seems to play a crucial role. Examples of codes such as orthogonal codes, biorthogonal codes, the $(24,12)$ extended Golay code, and the $(15,6)$ expurgated $\mathrm{BCH}$ code are discussed. The asymptotic coding gain at low signal-to-noise ratios is also studied.
\end{abstract}

Index Terms-Block codes, additive white Gaussian noise channel, low signal-to-noise ratios, error probabilities, coding gain.

\section{INTRODUCTION}

I $T$ IS WELL KNOWN that for binary block codes of a fixed rate using phase-shift keying modulation on an AWGN channel, for high signal-to-noise ratios, the decoder error probability is asymptotically controlled by the code's minimum distance: the higher the minimum distance, the better the code will perform. However, for some applications, for example when the code in question is the inner code in a concatenated coding system, it is important to know about decoder error probability at low signal-to-noise ratios. There is, however, surprisingly little published work on this problem. In [1], Posner studied the behavior of binary block codes on an AWGN channel at low signal-to-noise ratios, but most of his results are for "hard-decision" decoders, the only exception being his "soft-decision" results for orthogonal codes. In this paper,

Manuscript received November 12, 1990; revised February 25, 1992. The contributions of L. Swanson and E. Rodemich, and part of the contribution of R. McEliece, were done at the Jet Propulsion Laboratory, California Institute of Technology, under contract with the National Aeronautics and Space Administration. The contribution of C. Chao while he was a doctoral student at Caltech, and part of the contribution of R. McEliece, were supported by the Air Force Office of Scientific Research under Grant No. AFOSR-91-0037. This work was presented in part at the 1988 IEEE International Symposium on Information Theory, Kobe, Japan, June 1988 and in part at the 1990 IEEE International Symposium on Information Theory, San Diego, California, Jan. 14-19, 1990.

C.-C. Chao is with the Department of Electrical Engineering, National Tsing Hua University, Hsinchu, Taiwan 30043, R.O.C.

R. J. McEliece is with the Department of Electrical Engineering, California Institute of Technology, Pasadena, CA 91125.

L. Swanson and E. R. Rodemich are with the Jet Propulsion Laboratory, California Institute of Technology, Pasadena, CA 91109.

IEEE Log Number 9200886. which should be considered as a belated continuation of [1], we will study the decoder error probabilities for general binary block codes on an AWGN channel assuming maximum-likelihood, i.e., soft-decision, decoding.

Our approach is to study error probabilities of codes near the point where the bit signal-to-noise ratio $E_{b} / N_{0}$ is zero. One of the results obtained is the following approximation to the block-error probability:

$$
P_{E} \sim 1-\frac{1}{M}-\sqrt{\frac{E_{b}}{N_{0}}} \cdot \sqrt{\frac{R}{\pi}} \sum_{i=1}^{M-1} \sqrt{d_{i}} P_{i}
$$

where the binary block code considered has $M$ codewords and rate $R$, and $d_{i}$ is the Hamming distance between the $i$ th codeword $\boldsymbol{x}_{i}$ and the transmitted codeword $\boldsymbol{x}_{0}$. We shall see that the numbers $P_{i}$ in (1) depend in a complicated way on the global geometric structure of the code, so that it is apparently not possible to extract from (1) a simply-interpreted quantity that controls the code's error probability at low signal-to-noise ratios. Still, we shall see in several special cases, and conjecture in general, that the terms in (1) corresponding to codewords at minimum distance from $x_{0}$ dominate the expression, so that the minimum distance may still play a crucial role, even at low signal-to-noise ratios.

The full derivation of (1) is given in Section II. In Section III, a similar expression is found for the bit-error probability at low signal-to-noise ratios. The properties of $P_{i}$ are further explored in Section IV. Then the results are applied to examples such as orthogonal codes, biorthogonal codes, the $(24,12)$ extended Golay code and the $(15,6)$ expurgated BCH code in Section V. In Section VI, we study the asymptotic coding gain at low signal-to-noise ratios. Finally, discussions and conjectures are given in Section VII.

\section{BLOCK-ERROR ProBABILITY}

Let $\boldsymbol{C}=\left\{\boldsymbol{x}_{0}, \boldsymbol{x}_{1}, \cdots, \boldsymbol{x}_{M-1}\right\}$ be a binary block code (with components 0 and 1 ) of length $n$ and rate $R=$ $\left(\log _{2} M\right) / n$. We will evaluate the performance of $C$ on an unquantized AWGN channel as a function of the bit signal-to-noise ratio $E_{b} / N_{0}$, which we denote by $\lambda^{2}$. Suppose each codeword is equally likely to be selected for transmission. The codes we are interested in are all "sym- 
metric" in the sense that the error probabilities are independent of which codeword is transmitted (all linear codes have this property, for example). Therefore, we assume that $x_{0}$ is transmitted.

If $\hat{\boldsymbol{x}}_{0}$ is the counterpart of $\boldsymbol{x}_{0}$ with its components 0 changed to -1 , then the output of the channel becomes

$$
\boldsymbol{y}=\sqrt{S} \hat{x}_{0}+z,
$$

where the quantity $\sqrt{S}=\lambda \sqrt{2 R}$ and the vector $z=$ $\left(z_{1}, z_{2}, \cdots, z_{n}\right)$ has all components i.i.d. standard normal random variables (normal random variables with mean zero and variance 1). The maximum-likelihood decoder outputs the codeword with the minimum Euclidean distance from the received vector $\boldsymbol{y}$. This will be the correct decision, if and only if the decoded codeword was actually transmitted, or equivalently,

$$
|z|^{2}<\left|\boldsymbol{y}-\sqrt{S} \hat{\boldsymbol{x}}_{i}\right|^{2}, \quad \text { for } i=1,2, \cdots, M-1 .
$$

This inequality can be rewritten as

$$
\left\langle z, \sqrt{S}\left(\hat{\boldsymbol{x}}_{i}-\hat{\boldsymbol{x}}_{0}\right)\right\rangle<\frac{1}{2}\left|\sqrt{S}\left(\hat{\boldsymbol{x}}_{i}-\hat{\boldsymbol{x}}_{0}\right)\right|^{2},
$$

where $\left\langle z, \sqrt{S}\left(\hat{x}_{i}-\hat{x}_{0}\right)\right\rangle$ denotes the inner product of $z$ and $\sqrt{S}\left(\hat{\boldsymbol{x}}_{i}-\hat{\boldsymbol{x}}_{0}\right)$. Let $d_{i}$ be the Hamming distance between $x_{i}$ and $x_{0}$ and let $u_{i}$ be the vector in the direction of $\hat{\boldsymbol{x}}_{i}-\hat{\boldsymbol{x}}_{0}$ with magnitude $\sqrt{d_{i}}$. (Actually $\boldsymbol{u}_{i}$ is just $\boldsymbol{x}_{i}$ if $\boldsymbol{x}_{0}=\mathbf{0}$.) Then $\sqrt{S}\left(\hat{\boldsymbol{x}}_{i}-\hat{\boldsymbol{x}}_{0}\right)=2 \lambda \sqrt{2 R} \boldsymbol{u}_{i}$. If we define the normal random variables

$$
T_{i}=\left\langle z, u_{i}\right\rangle, \quad \text { for } i=1,2, \cdots, M-1,
$$

then $P_{C}$, the probability of correct decoding, is given by

$$
P_{C}=\operatorname{Pr}\left\{T_{i}<\lambda \sqrt{2 R} d_{i}, \text { for } i=1,2, \cdots, M-1\right\} .
$$

If the cumulative distribution function of $T_{1}, T_{2}, \cdots, T_{M-1}$ is denoted by $F\left(x_{1}, x_{2}, \cdots, x_{M-1}\right)$, then $P_{C}$ can be written as

$$
P_{C}=F\left(\lambda \sqrt{2 R} d_{1}, \lambda \sqrt{2 R} d_{2}, \cdots, \lambda \sqrt{2 R} d_{M-1}\right) .
$$

Note that $T_{1}, T_{2}, \cdots, T_{M-1}$ are $M-1$ normal random variables with mean zero and covariances

$$
\sigma_{i j}=\left\langle\boldsymbol{u}_{i}, \boldsymbol{u}_{j}\right\rangle \text {. }
$$

If $\boldsymbol{u}_{1}, \boldsymbol{u}_{2}, \cdots, \boldsymbol{u}_{M-1}$ are independent, then the covariance matrix $\boldsymbol{V}$ is nonsingular and the density function of $T_{1}, T_{2}, \cdots, T_{M-1}$ is given by

$$
p\left(x_{1}, x_{2}, \cdots, x_{M-1}\right)=\frac{1}{\sqrt{(2 \pi)^{M-1}|V|}} e^{-1 / 2 x^{T} V^{-1} x} .
$$

We can therefore write $P_{C}$ as an $M-1$-fold integral:

$$
P_{C}=\int_{-\infty}^{\lambda \sqrt{2 R} d_{1}} \int_{-\infty}^{\lambda \sqrt{2 R} d_{2}} \cdots \int_{-\infty}^{\lambda \sqrt{2} \bar{d} d_{M-1}} p(\boldsymbol{x}) d \boldsymbol{x} .
$$

However, if $\boldsymbol{u}_{1}, \boldsymbol{u}_{2}, \cdots, \boldsymbol{u}_{M-1}$ are not independent, then $\boldsymbol{V}$ is singular and $T_{1}, T_{2}, \cdots, T_{M-1}$ are "degenerate" in the sense of [2, p. 87], and we cannot convert $P_{C}$ to an integral. This is true for most practical codes because usually $M \gg n$. For example, the $(24,12)$ extended Golay code has $M=4096$ and $n=24$.

The approach we take is to view $P_{C}$ in (3) as a function of $\lambda$ and approximate $P_{C}$ by $P_{C}^{(0)}+\lambda P_{C}^{(1)}$, the first two terms in a power series expansion of $P_{C}(\lambda)$, in the neighborhood of $\lambda=0$. The following two theorems are essential to our derivation. Their proofs are given in Appendix A.

Theorem 1: Let $X_{1}, X_{2}, \cdots, X_{M-1}$ be $M-1$ mean zero jointly normal random variables (possibly degenerate), with covariances $\sigma_{i j}$ and with cumulative distribution function $F\left(x_{1}, x_{2}, \cdots, x_{M-1}\right)$, with every pair $X_{i}, X_{j}$ linearly independent (nondegenerate). Let $a_{1}, a_{2}, \cdots, a_{M-1}$ be nonnegative real numbers. Then, for $x>0$,

$F\left(a_{1} x, a_{2} x, \cdots, a_{M-1} x\right)=F(0,0, \cdots, 0)$

$$
+\frac{x}{\sqrt{2 \pi}} \sum_{i=1}^{M-1} \frac{a_{i}}{\sqrt{\sigma_{i i}}} P_{i}+O\left(x^{2}\right)
$$

where

$$
\begin{aligned}
& P_{i}=\operatorname{Pr}\left\{X_{j} \leq 0 \text { for } j \neq i \mid X_{i}=0\right\} \\
& \stackrel{\text { def }}{=} \lim _{h \rightarrow 0} \frac{\operatorname{Pr}\left\{X_{j} \leq 0 \text { for } j \neq i, 0<X_{i} \leq h\right\}}{\operatorname{Pr}\left\{0<X_{i} \leq h\right\}} .
\end{aligned}
$$

The next theorem establishes that the limit $P_{i}$ previously defined exists, and indeed establishes the rate at which the limit is approached.

Theorem 2: Define, for $h \geq 0$,

$$
P_{i}(h)=\operatorname{Pr}\left\{X_{j} \leq 0, \text { for } j \neq i \mid 0<X_{i} \leq h\right\} .
$$

Also define the random variables $Y_{j}$, for $j \neq i$, by

$$
Y_{j}=\sigma_{i i} X_{j}-\sigma_{i j} X_{i}
$$

Then,

$$
\lim _{h \rightarrow 0} P_{i}(h)=P_{i}=\operatorname{Pr}\left\{Y_{j} \leq 0, \text { for } j \neq i\right\}
$$

Also,

$$
\left|P_{i}(h)-P_{i}\right|=O(h) .
$$

Since no two $T_{i}$ 's are linearly independent because no two $u_{i}$ 's are, we can now use Theorems 1 and 2 to estimate $P_{C}$. Note that since the codes we consider are "symmetric,"

$$
F(0,0, \cdots, 0)=\frac{1}{M},
$$

which follows from the fact that each codeword is equally likely to be decoded if there is no signal at all. Also note that $\sigma_{i i}=E\left(T_{i}^{2}\right)=\left\langle\boldsymbol{u}_{i}, \boldsymbol{u}_{i}\right\rangle=d_{i}$. Hence, we have (combining (3) and Theorem 1)

$$
P_{C}=\frac{1}{M}+\lambda \cdot \sqrt{\frac{R}{\pi}} \sum_{i=1}^{M-1} \sqrt{d_{i}} P_{i}+O\left(\lambda^{2}\right),
$$

where $P_{i}$ is the conditional probability that $T_{1} \leq$ $0, \cdots, T_{i-1} \leq 0, T_{i+1} \leq 0, \cdots, T_{M-1} \leq 0$, given that $T_{i}=0$. 
The block-error probability $P_{E}=1-P_{C}$, and hence, for very small $\lambda$,

$$
P_{E} \sim 1-\frac{1}{M}-\lambda \cdot \sqrt{\frac{R}{\pi}} \sum_{i=1}^{M-1} \sqrt{d_{i}} P_{i},
$$

which is the result of (1).

\section{BIT-ERror ProbabILITY}

Maximum-likelihood decoding of binary linear block codes on an unquantized AWGN channel is now considered. We define $P_{b}$, the bit-error probability, to be the ratio of the expected number of information bits in error to the number of information bits. Let $\boldsymbol{C}=\left\{\boldsymbol{x}_{0}\right.$, $\left.\boldsymbol{x}_{1}, \cdots, x_{M-1}\right\}$ be a binary linear block code of length $n$ and rate $R=k / n$, where $M=2^{k}$. Assume $\boldsymbol{x}_{0}=\mathbf{0}$ is transmitted, and then

$$
\boldsymbol{y}=\sqrt{S} \hat{\boldsymbol{x}}_{0}+\boldsymbol{z}
$$

is the received vector. If the decoder chooses to output $\boldsymbol{x}_{i}$, then it will make $w_{i}$ bit errors, where $w_{i}$ is the number of 1 's in the information sequence corresponding to $x_{i}$. The expected number of information bits in error is hence

$$
b=\sum_{i=1}^{M-1} w_{i} \operatorname{Pr}\left\{\text { The decoder outputs } \boldsymbol{x}_{i} \cdot\right\},
$$

and the bit-error probability is $P_{b}=b / k$.

It remains to find the probability that the output codeword is $\boldsymbol{x}_{i}$. The maximum-likelihood decoder will output $\boldsymbol{x}_{i}$, if and only if the Euclidean distance between the received vector $\boldsymbol{y}$ and $\hat{\boldsymbol{x}}_{\boldsymbol{i}}$ is the smallest among all codewords, i.e.,

$$
\left|\boldsymbol{y}-\sqrt{S} \hat{\boldsymbol{x}}_{i}\right|<\left|\boldsymbol{y}-\sqrt{S} \hat{\boldsymbol{x}}_{j}\right|, \quad \text { for } j \neq i,
$$

which is equivalent to

$$
\left\langle z, \hat{\boldsymbol{x}}_{j}-\hat{\boldsymbol{x}}_{i}\right\rangle<\sqrt{S}\left\langle\hat{\boldsymbol{x}}_{0}, \hat{\boldsymbol{x}}_{i}-\hat{\boldsymbol{x}}_{j}\right\rangle, \quad \text { for } j \neq i .
$$

If we define $d_{i j}$ to be the Hamming distance between $\boldsymbol{x}_{i}$ and $\boldsymbol{x}_{j}$ and $\boldsymbol{u}_{i j}$ to be the vector in the direction of $\hat{\boldsymbol{x}}_{j}-\hat{\boldsymbol{x}}_{i}$ with magnitude $\sqrt{d_{i j}}$, then $\left\langle z, \hat{x}_{j}-\hat{x}_{i}\right\rangle=2\left\langle z, u_{i j}\right\rangle$. Also $\sqrt{S}\left\langle\hat{\boldsymbol{x}}_{0}, \hat{\boldsymbol{x}}_{i}-\hat{\boldsymbol{x}}_{j}\right\rangle=2 \lambda \sqrt{2 R}\left(d_{j}-d_{i}\right)$. Hence, (6) is equivalent to

$$
\left\langle z, \boldsymbol{u}_{i j}\right\rangle<\lambda \sqrt{2 R}\left(d_{j}-d_{i}\right), \quad \text { for } j \neq i .
$$

For $i \neq j$, we define the normal random variables $T_{i j}=$ $\left\langle\boldsymbol{z}, \boldsymbol{u}_{i j}\right\rangle$, which have mean zero and variances $d_{i j}$. Then, the bit-error probability is

$$
P_{b}=\frac{1}{k} \sum_{i=1}^{M-1} w_{i} \operatorname{Pr}\left\{T_{i j}<\lambda \sqrt{2 R}\left(d_{j}-d_{i}\right) \text {, for } j \neq i\right\} .
$$

Now we view $P_{b}$ as a function of $\lambda$ and approximate $P_{b}$ by $P_{b}^{(0)}+\lambda P_{b}^{(1)}$, the first two terms in a power series expansion of $P_{b}(\lambda)$ near $\lambda=0$. Proceeding as in the last section, we obtain

$$
P_{b}^{(0)}=\frac{1}{k} \sum_{i=1}^{M-1} w_{i} F^{i}(0,0, \cdots, 0),
$$

where $F^{i}$ is the cumulative distribution function of $T_{i 0}, \cdots, T_{i, i-1}, T_{i, i+1}, \cdots, T_{i, M-1}$. Also,

$$
P_{b}^{(1)}=\frac{1}{k} \sqrt{\frac{R}{\pi}} \sum_{i=1}^{M-1} w_{i} \sum_{j \neq i} \frac{\left(d_{j}-d_{i}\right)}{\sqrt{d_{i j}}} P_{i j},
$$

where $P_{i j}(i \neq j)$ is the conditional probability that $T_{i j^{\prime}} \leq$ 0 , for $j^{\prime} \neq i$ and $j^{\prime} \neq j$, given that $T_{i j}=0$. The linearity of the code is now used to simplify both the expressions of $P_{b}^{(0)}$ and $P_{b}^{(1)}$. For every pair of codewords $x_{i}, x_{j}$, there always exists another codeword $x_{l}$ such that $x_{i} \oplus x_{j}=x_{l}$, where $\oplus$ is the modulo- 2 addition. Since the normal distribution is symmetric about the origin, up to a permutation of the parameters, $F^{i}$, for $i=1,2, \cdots, M-1$ are equivalent to $F$ in the last section, and $P_{i j}=P_{l}$, where $x_{l}=x_{i} \oplus x_{j}$. By (4), it follows that

$$
P_{b}^{(0)}=\frac{1}{k M} \sum_{i=1}^{M-1} w_{i}=\frac{1}{k M} \cdot \frac{M k}{2}=\frac{1}{2},
$$

as expected, since each information bit is correct with probability $1 / 2$ when there is no signal. Now using the fact that $d_{i j}=d_{l}$ if $\boldsymbol{x}_{i} \oplus \boldsymbol{x}_{j}=\boldsymbol{x}_{l}$, we obtain

$$
P_{b} \sim \frac{1}{2}-\lambda \cdot \frac{1}{k} \sqrt{\frac{R}{\pi}} \sum_{i=1}^{M-1} w_{i} \sum_{\substack{j \neq i \\ \boldsymbol{x}_{i} \oplus x_{j}=x_{l}}} \frac{\left(d_{i}-d_{j}\right)}{\sqrt{d_{l}}} P_{l} .
$$

The previous approximation applies to all binary linear block codes. Further simplifications can be obtained if more assumptions are made. Now suppose $C$ is systematic and has the symmetry property such that each bit in the codeword is "permutationally equivalent" to each other bit, e.g., $C$ is cyclic, or more generally, its automorphism group (see definition in Section IV) contains a transitive permutation group. Then the bit-error probability can be found, alternatively, by dividing the expected number of codeword bits in error by the block length $n$. All the derivations remain the same as before except that $k$ and $w_{i}$ will now be replaced by $n$ and $d_{i}$, respectively. Then,

$$
P_{b} \sim \frac{1}{2}-\lambda \cdot \frac{1}{n} \sqrt{\frac{R}{\pi}} \sum_{i=1}^{M-1} d_{i} \sum_{\substack{j \neq i \\ \boldsymbol{x}_{i} \oplus \boldsymbol{x}_{j}=\boldsymbol{x}_{l}}} \frac{\left(d_{i}-d_{j}\right)}{\sqrt{d_{l}}} P_{l} .
$$

Algebraic manipulations show that

$$
\sum_{i=1}^{M-1} d_{i} \sum_{\substack{j \neq i \\ x_{i} \oplus \boldsymbol{x}_{j} \\=x_{l}}} \frac{\left(d_{i}-d_{j}\right)}{\sqrt{d_{l}}} P_{l}=\sum_{l=1}^{M-1} \frac{P_{l}}{\sqrt{d_{l}}} \sum_{i=0}^{M-1} d_{i}\left(d_{i}-d\left(\boldsymbol{x}_{i} \oplus \boldsymbol{x}_{l}\right)\right)
$$

where $d\left(\boldsymbol{x}_{i} \oplus \boldsymbol{x}_{l}\right)$ denotes the Hamming distance between $\boldsymbol{x}_{i} \oplus \boldsymbol{x}_{l}$ and $\boldsymbol{x}_{0}$. If we further assume that $C$ contains no repeated columns, i.e,. there are no two positions in the block where the corresponding bits are the same for all 
codewords, then from Appendix B,

$$
\sum_{i=1}^{M-1} d_{i}^{2}=n(n+1) 2^{k-2}
$$

and

$$
\sum_{i=1}^{M-1} d_{i} d\left(\boldsymbol{x}_{i} \oplus \boldsymbol{x}_{l}\right)=n(n+1) 2^{k-2}-d_{l} 2^{k-1} .
$$

Equation (8) can hence be written as

$$
P_{b} \sim \frac{1}{2}-\lambda \cdot \frac{M}{2 n} \sqrt{\frac{R}{\pi}} \sum_{i=1}^{M-1} \sqrt{d_{i}} P_{i} .
$$

Note that (1) and (11) have similar forms, and in particular that both involve the mysterious quantities $P_{i}$, which are the subject of the next section.

\section{PROPERTIES OF $P_{i}$}

The probability $P_{i}$ is the conditional probability that $T_{1} \leq 0, \cdots, T_{i-1} \leq 0, T_{i+1} \leq 0, \cdots, T_{M-1} \leq 0$, given that $T_{i}$ $=0$. In order to illustrate the calculation of $P_{i}$, consider the $M=4$ orthogonal code $\left(x_{0}=0000, x_{1}=0101, x_{2}=\right.$ $\left.0011, \boldsymbol{x}_{3}=0110\right\}$. By (2), $T_{1}=z_{2}+z_{4}, T_{2}=z_{3}+z_{4}, T_{3}=$ $z_{2}+z_{3}$, where $z_{1}, z_{2}, z_{3}, z_{4}$ are i.i.d. standard normal random variables. Therefore,

$$
\begin{aligned}
& P_{1}=\operatorname{Pr}\left\{T_{2} \leq 0, T_{3} \leq 0 \mid T_{1}=0\right\}, \\
& P_{2}=\operatorname{Pr}\left\{T_{1} \leq 0, T_{3} \leq 0 \mid T_{2}=0\right\}, \\
& P_{3}=\operatorname{Pr}\left\{T_{1} \leq 0, T_{2} \leq 0 \mid T_{3}=0\right\} .
\end{aligned}
$$

Since $z_{2}, z_{3}, z_{4}$ are i.i.d., it is easy to see that $P_{1}=P_{2}=P_{3}$. It remains to find the probability that $z_{3}+z_{4} \leq 0, z_{2}+z_{3}$ $\leq 0$, given that $z_{2}+z_{4}=0$, which is the conditional probability that a random point with a normal distribution in a three-dimensional space falls in the region described by $z_{3}+z_{4} \leq 0, z_{2}+z_{3} \leq 0$ given that it is on the plane $z_{2}+z_{4}=0$. We will see in the next section that $P_{1}=P_{2}$ $=P_{3}=\tan ^{-1} \sqrt{2} / \pi$. However, for most practical codes with $M \gg n$, it appears to be very difficult (if not impossible) to obtain a closed-form expression for $P_{i}$.

Definition 1: The set of coordinate permutations that map every codeword in the code $C$ into a (possibly different) codeword in $C$ is called the automorphism group of $\boldsymbol{C}$, denoted by Aut $(\boldsymbol{C})$.

It is known that $\operatorname{Aut}(C)$ is indeed a group. Automorphism groups of several block codes are discussed in [3]-[5]. There are computer search algorithms [6], [7] for finding the entire automorphism group of a code. Furthermore, the entire automorphism groups of all 2,3,4-error correcting binary primitive $\mathrm{BCH}$ codes have been determined algebraically in [8]. Note that the permutations in Aut $(C)$ partition the codewords in $C$ into equivalence classes. Codewords $\boldsymbol{x}_{i}$ and $\boldsymbol{x}_{j}$ are in the same equivalence class if there exists a permutation in $\operatorname{Aut}(C)$ that maps $\boldsymbol{x}_{i}$ to $\boldsymbol{x}_{j}$.

Theorem 3: Assume $\boldsymbol{x}_{0}=\mathbf{0}$. If $\boldsymbol{x}_{i}$ and $\boldsymbol{x}_{j}$ are in the same equivalence class partitioned by permutations in $\operatorname{Aut}(C)$, then $P_{i}=P_{j}$.

Proof: If $\boldsymbol{x}_{i}$ and $\boldsymbol{x}_{j}$ are in the same equivalence class, a permutation $\phi$ that maps $\boldsymbol{x}_{i}$ to $\boldsymbol{x}_{j}$ will map all the codewords other than $x_{0}$ and $x_{i}$ to codewords other than $\boldsymbol{x}_{0}$ and $\boldsymbol{x}_{j}$. It is impossible that tow different codewords are mapped to the same codeword because $\phi^{-1}$ is also in Aut $(C)$. Then, $T_{i}$ will be accordingly mapped to $T_{j}$ and $\left\{T_{l}: l \neq i\right\}$ to $\left\{T_{l}: l \neq j\right\}$, which implies that $P_{i}=P_{j}$.

In the preceding orthogonal code example, the permutation $\phi=(234)$ maps $x_{1}$ to $x_{3}, x_{3}$ to $x_{2}$ and $x_{2}$ to $x_{1}$, so $P_{1}=P_{2}=P_{3}$. For some practical codes, all the codewords of the same weight are in one equivalence class (but this is not generally true), so their corresponding $P_{i}$ 's are equal. Thus, the notation $P_{d}$ is used for all the codewords of weight $d$. For this case, (1) can be simplified to

$$
P_{E} \sim 1-\frac{1}{M}-\lambda \cdot \sqrt{\frac{R}{\pi}} \sum_{d} A_{d} \sqrt{d} P_{d},
$$

where $A(z)=\sum_{d} A_{d} z^{d}$ is the weight enumerator. Similarly, we can simplify (11) to

$$
P_{b} \sim \frac{1}{2}-\lambda \cdot \frac{M}{2 n} \sqrt{\frac{R}{\pi}} \sum_{d} A_{d} \sqrt{d} P_{d} .
$$

Recall that the original assumption for (11) is that $C$ is linear systematic with no repeated columns and Aut $(C)$ contains a transitive permutation group. ${ }^{1}$

Definition 2: Let $\boldsymbol{u}$ and $\boldsymbol{v}$ be binary vectors. If $\boldsymbol{u}$ has a 1 in every position that $v$ has a 1 , then we say that $\boldsymbol{u}$ covers $v$.

Theorem 4: Assume $\boldsymbol{x}_{0}=\mathbf{0}$. For a binary linear block code, if the codeword $\boldsymbol{x}_{i}$ covers a different nonzero codeword $\boldsymbol{x}_{j}$, then $P_{i}=0$.

Proof: Let $\boldsymbol{x}_{l}=\boldsymbol{x}_{i} \oplus \boldsymbol{x}_{j}$. Then, $\boldsymbol{x}_{l}$ is covered by $\boldsymbol{x}_{i}$ and $T_{i}=T_{j}+T_{l}$. The only case that both $T_{j}$ and $T_{l}$ are less than or equal to 0 given that $T_{i}=0$ is when $T_{j}=0$ and $T_{l}=0$. The theorem follows from the fact that all $T_{i}$ 's are continuous random variables.

For most practical codes, $M \gg n$, which means that there are many more random variables $T_{j}$ in the definition of the $P_{i}$ 's than the code dimension $n$. Hence, it is desirable to eliminate some redundant random variables $T_{j}$ to reduce the complexity of computing $P_{i}$. One simple result is that the condition $T_{l} \leq 0$ can be eliminated from $P_{i}$ if the codeword $\boldsymbol{x}_{l}$ covers another nonzero codeword $\boldsymbol{x}_{\dot{ }}$ with $T_{j} \leq 0$. This is proved by letting $\boldsymbol{x}_{m}=\boldsymbol{x}_{l} \oplus \boldsymbol{x}_{j}$, and then the conditions $T_{l}=T_{j}+T_{m}$ and $T_{j} \leq 0, T_{m} \leq 0$ guarantee that $T_{1} \leq 0$. The next theorem, which is proved in Appendix $\mathrm{C}$ by using the Farkas Alternative [9, p. 56] tells us in general how we can eliminate redundant $T_{j}$ 's. In

\footnotetext{
${ }^{1}$ A permutation group $G$ is transitive if, for any two symbols $i$ and $j$,
} there is a permutation $\phi \in G$ such that $i \phi=j$. 
the theorem, $\boldsymbol{A}$ is a matrix and $\boldsymbol{x}, \boldsymbol{y}, \boldsymbol{d}, \boldsymbol{b}$ are column vectors, and we say a vector $x \leq 0$ if all of its components $\leq 0$.

Theorem 5: Let the set $\mathscr{A}=\left\{\boldsymbol{x}: \boldsymbol{A X} \leq 0\right.$ and $\left.\boldsymbol{d}^{T} \boldsymbol{x}=0\right\}$ be nonempty. The inequality $\boldsymbol{b}^{T} \boldsymbol{x} \leq 0$ holds for all $\boldsymbol{x} \in \mathscr{A}$, if and only if $\boldsymbol{b}=\boldsymbol{A}^{T} \boldsymbol{y}+\alpha \boldsymbol{d}$, for some $\boldsymbol{y} \geq 0$ and $\alpha \in R$.

To interpret this theorem, we view each $T_{i} \leq 0$ as an inequality in $z_{1}, z_{2}, \cdots, z_{n}$. The theorem implies that given $T_{j} \leq 0$, for $j \neq i$, and $T_{i}=0$, the particular $T_{l} \leq 0$ is redundant and can be eliminated, if and only if $T_{l}=$ $\sum_{j \neq i, l} a_{j} T_{j}+\alpha T_{i}$, where $a_{j} \geq 0$ and $\alpha \in R$. Note that setting $\alpha=0$ reduces to the case stated previously. On the other hand, if we somehow want to create another redundant inequality $T_{l} \leq 0$, then $T_{l}$ must be in the form of $\sum_{j \neq i}^{M-1} a_{j} T_{j}+\alpha T_{i}$ with $a_{j} \geq 0$ and $\alpha \in R$.

Note that $\sigma_{i i}=\left\langle\boldsymbol{u}_{i}, \boldsymbol{u}_{i}\right\rangle=d_{i}$, and for codes with $\boldsymbol{x}_{0}=\mathbf{0}$, $\sigma_{i j}$ is the number of positions where $\boldsymbol{x}_{i}$ and $\boldsymbol{x}_{j}$ are both 1 . As previously mentioned, for most practical codes of interest, $M \gg n$; even after the redundant $T_{j}<0$ are eliminated by Theorem 5 , the number of remaining conditions is still very large compared with the code dimension $n$. Hence, it may be necessary to resort to Monte Carlo simulations to find approximate values for the $P_{i}$. Conditional probabilities are relatively difficult to work with, but Theorem 2 gives an alternate, unconditional, formula for the $P_{i}$ 's, which lends itself more easily to simulation:
(12) near $\lambda=0$, the block-error probability can be approximated by

$$
P_{E} \sim 1-\frac{1}{2^{k}}-\lambda \cdot\left(2^{k}-1\right) \sqrt{\frac{k}{2 \pi}} P_{2^{k-1}},
$$

where $P_{2^{k-1}}$ is for codewords of weight $2^{k-1}$. By using $P_{b}=\left(2^{k-1} P_{E}\right) /\left(2^{k}-1\right)[10$, p. 100$]$ or $(7)$, then

$$
P_{b} \sim \frac{1}{2}-\lambda \cdot 2^{k-1} \sqrt{\frac{k}{2 \pi}} P_{2^{k-1}}
$$

We now want to compute the value of $P_{2^{k-1}}$, which is the conditional probability that $T_{1} \leq 0, T_{2} \leq 0, \cdots, T_{2^{k}-2}$ $\leq 0$, given that $T_{2^{k}-1}=0$. By the structure of orthogonal codes, $T_{i}$, for $i=1,2, \cdots, 2^{k}-1$, are normal random variables with mean zero and covariances

$$
\sigma_{i j}= \begin{cases}2^{k-1}, & \text { if } i=j \\ 2^{k-2}, & \text { if } i \neq j\end{cases}
$$

An easy calculation verifies that the random variable $T_{i}$, for $i=1,2, \cdots, 2^{k}-1$, can be modeled by

$$
T_{i}=\sqrt{2^{k-2}}\left(X_{i}+X_{0}\right)
$$

where $X_{0}, X_{1}, \cdots, X_{2^{k}-1}$ are i.i.d. standard normal random variables. Hence,

$$
\begin{aligned}
P_{2^{k-1}} & =\lim _{h \rightarrow 0} \operatorname{Pr}\left\{X_{1}+X_{0} \leq 0, \cdots, X_{2^{k}-2}+X_{0} \leq 0 \mid 0<X_{2^{k}-1}+X_{0} \leq h\right\} \\
& =\lim _{h \rightarrow 0} \frac{\operatorname{Pr}\left\{X_{1} \leq-X_{0}, \cdots, X_{2^{k}-2} \leq-X_{0},-X_{0}<X_{2^{k}-1} \leq-X_{0}+h\right\}}{\operatorname{Pr}\left\{0<X_{2^{k}-1}+X_{0} \leq h\right\}} .
\end{aligned}
$$

$P_{i}=\operatorname{Pr}\left\{\sigma_{i i} T_{j}-\sigma_{i j} T_{i} \leq 0\right.$, for $\left.j \neq i\right\}$. First $n$ i.i.d. standard normal random variables $z_{i}$, for $i=1,2, \cdots, n$, are generated; then all necessary (nonredundant) conditions $\sigma_{i i} T_{j}$ $-\sigma_{i j} T_{i} \leq 0$ are tested. If all are satisfied, we record this event as a "success." If any one of the conditions fails, we record this event as a "failure." The procedure is repeated a large number of times; then the relative frequency of "success" will be an approximate value for $P_{i}$.

\section{EXAMPLES}

We now apply the results in previous sections to orthogonal codes, biorthogonal codes, the $(24,12)$ extended Golay code, and the $(15,6)$ expurgated $\mathrm{BCH}$ code.

\section{A. Orthogonal Codes}

We consider orthogonal codes with $M=2^{k}$ codewords, which are obtained from rows of $2^{k} \times 2^{k}$ normalized Hadamard matrices via the mapping that the +1 's are changed to 0 's and the - 1 's to 1's. Such Hadamard matrices can be constructed by the Sylvester method [3,p. 45]. All the nonzero codewords have weights $2^{k-1}$ and it is easy to see that they are in the same equivalence class. By
Since $X_{2^{k}-1}+X_{0}$ is normal with mean zero and variance $2, \operatorname{Pr}\left\{0<X_{2^{k}-1}+X_{0} \leq h\right\}=h / \sqrt{4 \pi}+O\left(h^{2}\right)$ as $h \rightarrow 0$. We also have

$$
\begin{aligned}
\lim _{h \rightarrow 0} \operatorname{Pr}\left\{X_{1} \leq-X_{0}, \cdots, X_{2^{k}-2} \leq-X_{0},-X_{0}\right. \\
\left.<X_{2^{k}-1} \leq-X_{0}+h\right\} \\
=\lim _{h \rightarrow 0} \int_{-\infty}^{\infty} h \cdot Z(-t)[P(-t)]^{2^{k}-2} Z(t) \cdot d t+O\left(h^{2}\right) \\
\quad=\lim _{h \rightarrow 0} \frac{h}{\sqrt{2 \pi}} \int_{-\infty}^{\infty} Z(\sqrt{2} t)[P(t)]^{2^{k}-2} d t+O\left(h^{2}\right),
\end{aligned}
$$

where $Z(x)=e^{-x^{2} / 2} / \sqrt{2 \pi}$ and $P(x)=\int_{-\infty}^{x} Z(t) d t$. Finally, we obtain

$$
P_{2^{k-1}}=\sqrt{2} \int_{-\infty}^{\infty} Z(\sqrt{2} t)[P(t)]^{2^{k}-2} d t .
$$

The same result was obtained in [1] by directly expanding into a power series the expressions of the error probabilities for orthogonal codes from [11]. Our $P_{2^{k-1}}$ is equal to $\sqrt{2} A_{2^{k}-1}$ in the notation of [1]. In particular, for $k=2$, $A_{3}$ was shown to be $\tan ^{-1} \sqrt{2} /(\pi \sqrt{2})$; it follows that $P_{2}=\tan ^{-1} \sqrt{2} / \pi$. Since it was shown that $A_{v} \approx$ 
$\left(2 / \nu^{2}\right) \sqrt{\pi \ln \nu}$ for large $\nu$,

$$
P_{2^{k-1}} \approx \frac{2}{\left(2^{k}-1\right)^{2}} \sqrt{2 \pi \ln \left(2^{k}-1\right)}, \quad \text { for large } k
$$

Equation (16) has been integrated numerically for $k=2$ to 10 , and the results are listed in Table $\mathrm{I}$, and so are the quantities $\left(2^{k}-1\right) \sqrt{k /(2 \pi)} \mathrm{P}_{2^{k-1}}$ and $2^{k-1}$ $\sqrt{k /(2 \pi)} P_{2^{k-1}}$, which are the key elements of (14) and (15), respectively. Note that, for orthogonal codes at very low signal-to-noise ratios, the bit-error probability increases with $k$, or the number of codewords $M$.

\section{B. Biorthogonal Codes}

A biorthogonal code defined here consists of the codewords of an orthogonal code and their complements. We consider biorthogonal codes with $M=2^{k}, k \geq 2$, codewords. The biorthogonal code is the first-order Reed-Muller code if the corresponding orthogonal code is obtained by the Sylvester construction [3, p. 373]. All the codewords except the all-zero and all-one codewords have weights $2^{k-2}$.

Proposition 1: All the codewords except the all-zero and all-one codewords in a biorthogonal code are in one equivalence class.

Proof: For a biorthogonal code with $M=2^{k}, k \geq 2$, there are $2^{k}-2$ codewords of weight $2^{k-2} ; 2^{k-1}-1$ of them begin with 0 because they are codewords of an orthogonal code, and the rest begin with 1 . Since all the nonzero codewords in an orthogonal code are in one equivalence class, it follows that there are at most two equivalence classes for codewords of weight $2^{k-2}$ in a biorthogonal code, one for each half. However, since the automorphism group of a Reed-Muller code contains the general affine group that is triply transitive ${ }^{2}[3, \mathrm{pp}$. 398-400], there exist permutations in the automorphism group of a biorthogonal code (which is the first-order Reed-Muller code) that map a nonzero codeword beginning with 0 to a codeword beginning with 1 . The proposition hence follows.

The all-one codeword covers every codeword of weight $2^{k-2}$, so from Theorem 4 the corresponding $P_{i}$ is zero. By (12), we now have

$$
P_{E} \sim 1-\frac{1}{2^{k}}-\lambda \cdot\left(2^{k}-2\right) \sqrt{\frac{k}{2 \pi}} P_{2^{k-2}} .
$$

Since a biorthogonal code contains no repeated columns, can be encoded as a systematic code, and its automor-

\footnotetext{
${ }^{2}$ A permutation group $G$ is $t$-fold transitive if, given $t$ distinct symbols $i_{1}, i_{2}, \cdots, i_{t}$, and $t$ distinct symbols $j_{1}, j_{2}, \cdots, j_{t}$, there is a permutation $\phi \in G$ such that $i_{1} \phi=j_{1}, i_{2} \phi=j_{2}, \cdots, i_{t} \phi=j_{t}$.
}

TABLE I

\begin{tabular}{|c|c|c|c|}
\hline$k$ & $P_{2^{k-1}}$ & $\left(2^{k}-1\right) \sqrt{k /(2 \pi)} P_{2^{k-1}}$ & $2^{k-1} \sqrt{k /(2 \pi)} P_{2^{k-1}}$ \\
\hline 2 & $3.0409 e-1$ & $5.1469 e-1$ & $3.4312 e-1$ \\
\hline 3 & $9.0117 e-2$ & $4.3589 e-1$ & $2.4908 e-1$ \\
\hline 4 & $2.6084 e-2$ & $3.1219 e-1$ & $1.6650 e-1$ \\
\hline 5 & $7.3959 e-3$ & $2.0453 e-1$ & $1.0556 e-1$ \\
\hline 6 & $2.0606 e-3$ & $1.2686 e-1$ & $6.4436 e-2$ \\
\hline 7 & $5.6580 e-4$ & $7.5845 e-2$ & $3.8221 e-2$ \\
\hline 8 & $1.5351 e-4$ & $4.4170 e-2$ & $2.2171 e-2$ \\
\hline 9 & $4.1242 e-5$ & $2.5222 e-2$ & $1.2636 e-2$ \\
\hline 10 & $1.0991 e-5$ & $1.4185 e-2$ & $7.0995 e-3$ \\
\hline
\end{tabular}

$P_{2^{k-1}}$ for Orthogonal Codes

TABLE II

$P_{2^{k-2}}$ FOR BIORTHOGONAL CODES

\begin{tabular}{rcc}
\hline$k$ & $P_{2^{k-2}}$ & $\left(2^{k}-2\right) \sqrt{k /(2 \pi)} P_{2^{k-2}}$ \\
\hline 3 & $1.0817 e-1$ & $4.4848 e-1$ \\
4 & $2.8223 e-2$ & $3.1526 e-1$ \\
5 & $7.6703 e-3$ & $2.0527 e-1$ \\
6 & $2.0968 e-3$ & $1.2704 e-1$ \\
7 & $5.7062 e-4$ & $7.5889 e-2$ \\
8 & $1.5415 e-4$ & $4.4180 e-2$ \\
9 & $4.1327 e-5$ & $2.5225 e-2$ \\
10 & $1.1003 e-5$ & $1.4186 e-2$ \\
11 & $2.9114 e-6$ & $7.8817 e-3$ \\
\hline
\end{tabular}

phism group contains a triply transitive group, by (13) for $\lambda$ near 0 ,

$$
P_{b} \sim \frac{1}{2}-\lambda \cdot\left(2^{k}-2\right) \sqrt{\frac{k}{2 \pi}} P_{2^{k-2}}
$$

Now, our goal is to find an analytical expression for $P_{2^{k-2}}$, the conditional probability that $T_{2} \leq 0, T_{3} \leq$ $0, \cdots, T_{2^{k}-1} \leq 0$, given that $T_{1}=0$. (Here, we number the codewords in such a way that $x_{i}$, for $i=0,1, \cdots, 2^{k-1}-1$, are codewords of a corresponding orthogonal code and $\boldsymbol{x}_{2^{k-1}+i}$, for $i=0,1, \cdots, 2^{k-1}-1$, are the complements of $\boldsymbol{x}_{i}$.) Since the all-one codeword $\boldsymbol{x}_{2^{k-1}}$ covers every codeword of weight $2^{k-2}$, the condition $T_{2^{k-1}} \leq 0$ is redundant and can be discarded. From the structure of biorthogonal codes, the covariances between $T_{i}$ and $T_{j}$, for $i, j=$ $1, \cdots, 2^{k-1}-1,2^{k-1}+1, \cdots, 2^{k}-1$, are given by

$$
\sigma_{i j}= \begin{cases}2^{k-2}, & \text { if } i=j \\ 0, & \text { if }|i-j|=2^{k-1} \\ 2^{k-3}, & \text { otherwise. }\end{cases}
$$

We then model the random variables $T_{1}, \cdots, T_{2^{k-1}-1}$, $T_{2^{k-1}+1}, \cdots, T_{2^{k}-1}$ by

$$
T_{i}=\sqrt{2^{k-3}}\left(X_{0}+X_{i}\right)
$$

and

$$
T_{2^{k-1}+i}=\sqrt{2^{k-3}}\left(X_{0}-X_{i}\right),
$$

where $i=1,2, \cdots, 2^{k-1}-1$ and $X_{0}, X_{1}, \cdots, X_{2^{k}-1}$ are i.i.d. 
standard normal random variables. Thus,

and the bit-error probability can be approximated by

$$
\begin{aligned}
P_{2^{k-2}}= & \lim _{h \rightarrow 0} \operatorname{Pr}\left\{X_{0}-X_{1} \leq 0 \text { and } X_{0}+X_{i} \leq 0, X_{0}-X_{i} \leq 0, \text { for } i=2,3, \cdots, 2^{k-1}-10<X_{0}+X_{1} \leq h\right\} \\
= & \lim _{h \rightarrow 0} \operatorname{Pr}\left\{X_{0} \leq 0,-X_{0}<X_{1} \leq-X_{0}+h, \text { and } X_{0} \leq X_{i} \leq-X_{0},\right. \\
& \text { for } \left.i=2,3, \cdots, 2^{k-1}-1\right\} / \operatorname{Pr}\left\{0<X_{0}+X_{1} \leq h\right\} \\
= & \lim _{h \rightarrow 0} \frac{\int_{-x}^{0} h \cdot Z(-t)[P(-t)-P(t)]^{2^{k-1}-2} Z(t) d t+O\left(h^{2}\right)}{h / \sqrt{4 \pi}+O\left(h^{2}\right)} \\
= & \sqrt{2} \int_{0}^{\infty} Z(\sqrt{2} t)[P(t)-P(-t)]^{2^{k-1}-2} d t .
\end{aligned}
$$

The same result can be obtained if we expand into a power series in $\lambda$ the expressions for error probabilities in [11]. We have integrated numerically the expressions for $P_{2^{k-2}}, k=3,4, \cdots, 11$, and listed the results in Table II, along with the quantities $\left(2^{k}-2\right) \sqrt{k /(2 \pi)} P_{2^{k-2}}$. Again note that for biorthogonal codes at very low signal-to-noise ratios the bit-error probability increases with the number of codewords.

\section{The $(24,12)$ Extended Golay Code}

The $(24,12)$ extended Golay code is obtained by adding an overall parity check bit to the perfect triple-errorcorrecting $(23,12)$ Golay code. Its weight enumerator is $A(z)=1+759 z^{8}+2576 z^{12}+759 z^{16}+z^{24}$. Note that the codeword of weight 24 is the all-one codeword, which covers all other nonzero codewords. The automorphism group of the $(24,12)$ Golay code is the Mathieu group $M_{24}$ which is five-fold transitive [3, pp. 636-641].

Proposition 2 [3, p. 638]: All the codewords of weight 8 are in one equivalence class.

Proposition 3 [3, p. 641]: All the codewords of weight 12 are in one equivalence class.

Proposition 4: All the codewords of weight 16 are in one equivalence class.

Proof: The permutation that maps one codeword to another codeword will do the same to their complements. Since the complement of any codeword of weight 8 is a codeword of weight 16 and vice versa, the proposition follows from Proposition 2.

Proposition 5: Every codeword of weight 16 covers codewords of weight 8 .

Proof: By Proposition 4, this proposition can be proved by finding an instance that a codeword of weight 16 covers a codeword of weight 8 . This is easily done by inspecting a generator matrix for the $(24,12)$ extended Golay code, e.g., the one in $[3$, p. 65$]$.

Now, near $\lambda=0$ the block-error probability can be approximated by

$$
P_{E} \sim \frac{4095}{4096}-\lambda \cdot \sqrt{\frac{1}{2 \pi}}\left(759 \sqrt{8} P_{8}+2576 \sqrt{12} P_{12}\right),
$$

$$
P_{b} \sim \frac{1}{2}-\lambda \cdot \frac{256}{3} \sqrt{\frac{1}{2 \pi}}\left(759 \sqrt{8} P_{8}+2576 \sqrt{12} P_{12}\right) .
$$

Note that the codewords of weight 16 and 24 play no part in the approximations for $P_{E}$ and $P_{b}$, neither do they in $P_{8}$ and $P_{12}$. Unlike the last two examples, we do not expect exact analytical expressions for $P_{8}$ and $P_{12}$. The procedure described in the last section is used to simulate $P_{8}$ and $P_{12}$. All the inequalities corresponding to the all-one codeword and codewords of weight 16 can be discarded because of Theorem 5 . The results are $P_{8} \approx 4.0$ $\times 10^{-6}$ and $P_{12} \approx 4 \times 10^{-8}$. (Since $P_{12}$ is very small, the reliability of the value obtained is doubtful but the magnitude should be correct.) Then,

$$
\begin{aligned}
P_{E} & \sim \frac{4095}{4096}-\lambda \cdot \sqrt{\frac{1}{2 \pi}}\left(8.6 \times 10^{-3}+3.6 \times 10^{-4}\right) \\
& \approx \frac{4095}{4096}-\lambda \cdot\left(3.6 \times 10^{-3}\right),
\end{aligned}
$$

and

$$
\begin{aligned}
P_{b} & \sim \frac{1}{2}-\lambda \cdot \frac{256}{3} \sqrt{\frac{1}{2 \pi}}\left(8.6 \times 10^{-3}+3.6 \times 10^{-4}\right) \\
& \approx \frac{1}{2}-\lambda \cdot(0.30) .
\end{aligned}
$$

Note that the terms above for codewords of weight 8 (codewords at the minimum distance) are much larger than the terms for $P_{12}$, so that in a sense, the behavior of the code at low signal-to-noise ratios is controlled by the minimum distance.

\section{The $(15,6)$ Expurgated BCH Code}

We now consider the $(15,6)$ expurgated $\mathrm{BCH}$ code with generator polynomial $\left(x^{4}+x+1\right)\left(x^{4}+x^{3}+x^{2}+\mathrm{x}+\right.$ $1)(x+1)=x^{9}+x^{6}+x^{5}+x^{4}+x+1$. Its weight enumerator is $A(z)=1+30 z^{6}+15 z^{8}+18 z^{10}$. It is known 
[7], [8] that the complete automorphism group of the $(15,7) \mathrm{BCH}$ code with $d_{\min }=5$ is the group $\left\{b x^{2^{i}}+\right.$ $b^{\prime} x^{2^{i+2}}: b, b^{\prime} \in G F\left(2^{4}\right), b^{2^{2+1}} \neq b^{2^{2}+1}$ and $\left.i=0,1\right\}$. We also know that the automorphism group of an expurgated code contains that of the original code. By examining the codewords of the $(15,6)$ expurgated $\mathrm{BCH}$ code, it can then be shown that all the codewords of equal weight are in the same equivalence classes. Therefore, the error probabilities near $\lambda=0$ are approximately

$$
P_{E} \sim \frac{63}{64}-\lambda \cdot \sqrt{\frac{2}{5 \pi}}\left(30 \sqrt{6} P_{6}+15 \sqrt{8} P_{8}+18 \sqrt{10} P_{10}\right),
$$

and

$$
P_{b} \sim \frac{1}{2}-\lambda \cdot \frac{32}{15} \sqrt{\frac{2}{5 \pi}}\left(30 \sqrt{6} P_{6}+15 \sqrt{8} P_{8}+18 \sqrt{10} P_{10}\right) .
$$

From a Monte Carlo simulation, $P_{6} \approx 4.1 \times 10^{-3}, P_{8} \approx$ $8.9 \times 10^{-4}$ and $P_{10} \approx 9.4 \times 10^{-5}$. Thus,

$$
\begin{aligned}
P_{E} & \sim \frac{63}{64}-\lambda \cdot \sqrt{\frac{2}{5 \pi}}\left(0.30+3.8 \times 10^{-2}+5.4 \times 10^{-3}\right) \\
& \approx \frac{63}{64}-\lambda \cdot(0.12),
\end{aligned}
$$

and

$$
P_{b} \sim \frac{1}{2}-\lambda \cdot(0.26)
$$

Note again that the terms above for codewords at the minimum distance are much larger than the remaining terms.

\section{Asymptotic Coding Gain}

The coding gain is the ratio of the signal-to-noise ratio without coding to the signal-to-noise ratio required when using an error-correcting code to achieve the same error probability. We define the asymptotic coding gain as the limit, as the signal-to-noise ratio approaches zero, of the coding gain. Two theorems based on the criteria of $P_{E}$ and $P_{b}$, respectively, will be given.

We now derive approximations to $P_{E}$ and $P_{b}$ at low signal-to-noise ratios when no coding is used. For an unquantized AWGN channel, the bit-error probability without coding is

$$
P_{b}=Q(\sqrt{2} \lambda),
$$

where $Q(x)=\int_{x}^{\infty} e^{-t^{2} / 2} / \sqrt{2 \pi} d t$. Thus, near $\lambda=0$,

$$
P_{b} \sim \frac{1}{2}-\lambda \cdot \frac{1}{\sqrt{\pi}} .
$$

If $k$ bits are grouped as a block without coding, a block error occurs when there is at least one erroneous bit and so

$$
P_{E}=1-\left(1-P_{b}\right)^{k},
$$

which gives the following approximation near $\lambda=0$ :

$$
P_{E} \sim 1-\frac{1}{2^{k}}-\lambda \cdot \frac{k}{2^{k-1} \sqrt{\pi}} .
$$

Comparing (1), (7), (11), (17), and (18), we obtain the following theorems.

Theorem 6: For binary block codes, with the criterion based on block-error probability, the asymptotic coding gain at low signal-to-noise ratios is given by

$$
G_{E}=\frac{2^{2(k-1)}}{n k}\left(\sum_{i=1}^{M-1} \sqrt{d_{i}} P_{i}\right)^{2}
$$

Theorem 7: For binary linear block codes, with the criterion based on bit-error probability, the asymptotic coding gain at low signal-to-noise ratios is given by

$$
G_{b}=\frac{1}{n k}\left(\sum_{i=1}^{M-1} w_{i} \sum_{\substack{j \neq i \\ x_{i} \oplus x_{j}=x_{l}}} \frac{\left(d_{i}-d_{j}\right)}{\sqrt{d_{l}}} P_{l}\right)^{2}
$$

If the code used is systematic with no repeated columns and its automorphism group contains a transitive permutation group, then the asymptotic coding gain can be simplified to

$$
G_{b}=\frac{k}{n}\left(\frac{2^{k-1}}{n} \sum_{i=1}^{M-1} \sqrt{d_{i}} P_{i}\right)^{2},
$$

which is equal to $(k / n)^{2} G_{E}$.

We now apply the results in Theorems 6 and 7 to the codes discussed in Section V. For orthogonal codes with $2^{k}$ codewords, based on the $P_{E}$-criterion, the asymptotic coding gain is

$$
G_{E}=\frac{2^{2(k-1)}\left(2^{k}-1\right)^{2}}{k}\left(\int_{-\infty}^{\infty} Z(\sqrt{2} t)[P(t)]^{2^{k}-2} d t\right)^{2},
$$

which approaches $\pi \ln 2 \approx 3.38 \mathrm{~dB}$ as $k \rightarrow \infty$. For the $P_{b}$-criterion, the asymptotic coding gain becomes

$$
G_{b}=k 2^{2(k-1)}\left(\int_{-\infty}^{\infty} Z(\sqrt{2} t)[P(t)]^{2^{k}-2} d t\right)^{2},
$$

which is asymptotic in $k$ to $(\pi \ln 2) k^{2} / 2^{2 k}$. The same results were obtained in [1]. The asymptotic coding gains based on criterions $P_{E}$ and $P_{b}$ for orthogonal codes are listed in Table III. Note that, based on the $P_{E}$-criterion, except for $k=2$, orthogonal codes result in positive coding gain compared with no coding at low signal-to-noise ratios and the gain increases with the number of codewords. However, for the $P_{b}$-criterion, there is always a coding loss when using an orthogonal code and the loss increases with $k$.

For biorthogonal codes with $2^{k}$ codewords, based on the $P_{E}$-criterion,

$G_{E}=\frac{2^{2(k-1)}\left(2^{k}-2\right)^{2}}{k}$

$$
\cdot\left(\int_{0}^{\infty} Z(\sqrt{2} t)[P(t)-P(-t)]^{2^{k-1}-2} d t\right)^{2}
$$


TABLE III

Asymptotic CODING GAIN FOR ORTHOGONAL CODES

\begin{tabular}{ccc}
\multicolumn{3}{c}{ ASYMPTOTIC CODING GAIN FOR ORTHOGONAL CODES } \\
\hline$k$ & $G_{E}$ & $G_{b}$ \\
\hline 2 & $-0.798 \mathrm{~dB}$ & $-4.32 \mathrm{~dB}$ \\
3 & $0.258 \mathrm{~dB}$ & $-7.10 \mathrm{~dB}$ \\
4 & $0.880 \mathrm{~dB}$ & $-10.6 \mathrm{~dB}$ \\
5 & $1.29 \mathrm{~dB}$ & $-14.6 \mathrm{~dB}$ \\
6 & $1.58 \mathrm{~dB}$ & $-18.8 \mathrm{~dB}$ \\
7 & $1.79 \mathrm{~dB}$ & $-23.4 \mathrm{~dB}$ \\
8 & $1.96 \mathrm{~dB}$ & $-28.1 \mathrm{~dB}$ \\
9 & $2.09 \mathrm{~dB}$ & $-33.0 \mathrm{~dB}$ \\
10 & $2.19 \mathrm{~dB}$ & $-38.0 \mathrm{~dB}$ \\
\hline
\end{tabular}

For the $P_{b}$-criterion,

$$
G_{b}=k\left(2^{k}-2\right)^{2}\left(\int_{0}^{\infty} Z(\sqrt{2} t)[P(t)-P(-t)]^{2^{k-1}-2} d t\right)^{2} .
$$

The asymptotic coding gains for biorthogonal codes are tabulated in Table IV. It is observed that with the $P_{E^{-}}$ criterion, there is a positive coding gain when using a biorthogonal code and the gain increases with the number of codewords. Again, with the $P_{b}$-criterion, there is always a coding loss and the loss increases with $k$.

For the $(24,12)$ extended Golay code,

$$
\begin{aligned}
G_{E} & =\frac{2^{22}}{24 \cdot 12}\left(759 \sqrt{8} P_{8}+2576 \sqrt{12} P_{12}\right)^{2} \\
& \approx 1.16 \approx 0.66 \mathrm{~dB},
\end{aligned}
$$

which is a gain over no coding. Also

$$
G_{b}=\left(\frac{k}{n}\right)^{2} G_{E} \approx 0.291 \approx-5.3 \mathrm{~dB},
$$

which is a loss. For the $(15,6)$ expurgated $\mathrm{BCH}$ code,

$$
\begin{aligned}
G_{E} & =\frac{2^{10}}{15 \cdot 6}\left(30 \sqrt{6} P_{6}+15 \sqrt{8} P_{8}+18 \sqrt{10} P_{10}\right)^{2} \\
& \approx 1.35 \approx 1.3 \mathrm{~dB},
\end{aligned}
$$

and

$$
G_{b}=\left(\frac{k}{n}\right)^{2} G_{E} \approx 0.216 \approx-6.6 \mathrm{~dB} .
$$

It was shown in [1] that if hard quantization is used on an AWGN channel, using the bit-error probability criterion, any coding scheme results in a loss at low signal-tonoise ratios. Note that for all the codes discussed in the last section, based on the $P_{b}$-criterion, there is a loss with respect to no coding. We conjecture that this is true for binary codes in general on an unquantized AWGN channel with maximum-likelihood decoding. Since, at low signal-to-noise ratios, maximum-likelihood decoding is not the scheme that minimizes the bit-error probability, a stronger conjecture is that, based on the $P_{b}$-criterion, any coding scheme will result in a loss on an unquantized AWGN channel at sufficiently low signal-to-noise ratios.

\section{CONCLUDING REMARKS}

In this paper, we have derived error probabilities and asymptotic coding gains of binary block codes used on an
TABLE IV

Asymptotic Coding GAIN For Biorthogonal Codes

\begin{tabular}{rlc}
\hline$k$ & $G_{E}$ & $G_{b}$ \\
\hline 3 & $0.505 \mathrm{~dB}$ & $-1.99 \mathrm{~dB}$ \\
4 & $0.965 \mathrm{~dB}$ & $-5.06 \mathrm{~dB}$ \\
5 & $1.32 \mathrm{~dB}$ & $-8.78 \mathrm{~dB}$ \\
6 & $1.59 \mathrm{~dB}$ & $-12.9 \mathrm{~dB}$ \\
7 & $1.80 \mathrm{~dB}$ & $-17.4 \mathrm{~dB}$ \\
8 & $1.96 \mathrm{~dB}$ & $-22.1 \mathrm{~dB}$ \\
9 & $2.09 \mathrm{~dB}$ & $-27.0 \mathrm{~dB}$ \\
10 & $2.19 \mathrm{~dB}$ & $-32.0 \mathrm{~dB}$ \\
11 & $2.28 \mathrm{~dB}$ & $-37.1 \mathrm{~dB}$ \\
\hline
\end{tabular}

unquantized AWGN channel at very low signal-to-noise ratios. The results show that the performance depends heavily on the codes' global geometric structures through the important quantity $P_{i}$ for each codeword. Since the computation of $P_{i}$ involves degenerate multivariate normal random variables, for most cases it is very difficult to get closed-form expressions and we do not expect such exist. In order to have better insight to the performance of codes at low signal-to-noise ratios, we suggest further research in finding tight lower and upper bounds for $P_{i}$.

From the results of this paper, we know that the codewords which cover other codewords do not affect the low signal-to-noise ratio performance at all. We conjecture that for $\boldsymbol{x}_{i}, \boldsymbol{x}_{j} \in C$, if $d_{i}<d_{j}$, then $P_{i}>P_{j}$. Now consider the quantity $\sum_{i=1}^{M-1} \sqrt{d_{i}} P_{i}$, which plays an important part in the expressions of error probabilities. It is observed that the term $\sqrt{d_{i}} P_{i}$ at the code's minimum distance is larger than the sum of remaining terms. We also conjecture that it is true in general.

One may wonder how the approximations obtained for the block-error probability and bit-error probability compare to the exact performances at low signal-to-noise ratios. An example is shown in Fig. 1, where the exact block-error probability and the approximation obtained in the paper for the biorthogonal code with $M=16$ codewords on an unquantized AWGN channel are plotted as functions of the bit signal-to-noise ratio $E_{b} / N_{0}$. As seen from the figure, the approximation is very tight for $E_{b} / N_{0}$ up to $-10 \mathrm{~dB}$ and not bad up to $-5 \mathrm{~dB}$.

\section{APPENDIX A}

PROOFS OF THEOREMS 1 AND 2

Theorem 1: Let $X_{1}, X_{2}, \cdots, X_{M-1}$ be $M-1$ mean zero jointly normal random variables (possibly degenerate), with covariances $\sigma_{i j}$ and with cumulative distribution function $F\left(x_{1}, x_{2}, \cdots, x_{M-1}\right)$, with every pair $X_{i}, X_{j}$ linearly independent (nondegenerate). Let $a_{1}, a_{2}, \cdots, a_{M-1}$ be nonnegative real numbers. Then, for $x>0$,

$$
\begin{aligned}
F\left(a_{1} x, a_{2} x, \cdots, a_{M-1} x\right)= & F(0,0, \cdots, 0) \\
& +\frac{x}{\sqrt{2 \pi}} \sum_{i=1}^{M-1} \frac{a_{i}}{\sqrt{\sigma_{i i}}} P_{i}+O\left(x^{2}\right),
\end{aligned}
$$

where

$$
P_{i}=\operatorname{Pr}\left\{X_{j} \leq 0 \text { for } j \neq i \mid X_{i}=0\right\}
$$




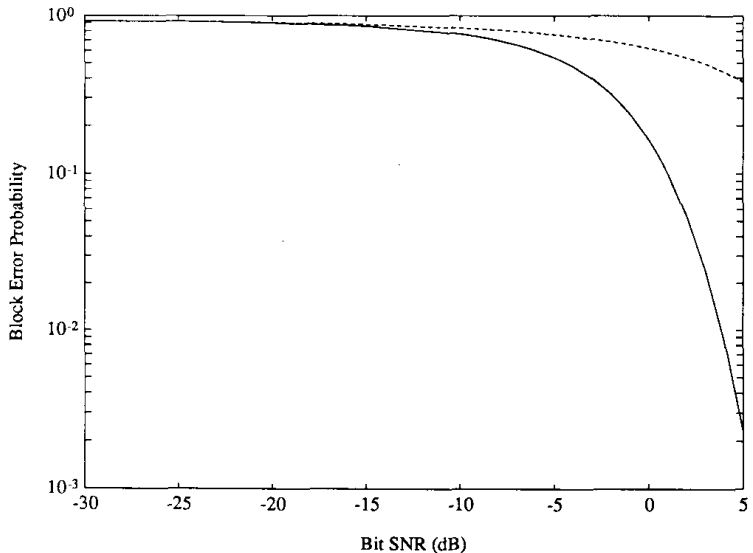

Fig. 1. Block-error probability of the biorthogonal code with $M=16$ codewords on an unquantized AWGN channel. (Solid line: exact performance. Dashed line: approximation.)

$$
\stackrel{\text { def }}{=} \lim _{h \rightarrow 0} \frac{\operatorname{Pr}\left\{X_{j} \leq 0 \text { for } j \neq i, 0<X_{i} \leq h\right\}}{\operatorname{Pr}\left\{0<X_{i} \leq h\right\}} .
$$

Proof: We partition the event $\left\{X_{1} \leq a_{1} x, \cdots, X_{M-1} \leq\right.$ $a_{M-1} x$ into $2^{M-1}$ subsets, according to which of the $X_{i}$, s are positive. If none of them are positive, we get the term $F(0,0, \cdots, 0)$ in (19). If two or more of the $X_{i}$ 's are positive, say $X_{i}>0$ and $X_{j}>0$, then the corresponding probability is $\leq \operatorname{Pr}\left\{0<X_{i} \leq\right.$ $a_{i} x, 0<X_{j} \leq a_{j} x$, which, since we assume that $X_{i}$ and $X_{j}$ are linearly independent, satisfies

$$
\operatorname{Pr}\left\{0<X_{i} \leq a_{i} x, 0<X_{j} \leq a_{j} x\right\}=O\left(x^{2}\right),
$$

so that the sum of the terms with at least two positive $X_{i}$ 's is $O\left(x^{2}\right)$. Finally we come to the terms with exactly one positive $X_{i}$. A typical term of this form is

$$
\begin{aligned}
& \operatorname{Pr}\left\{X_{j} \leq 0 \text { for } j \neq i, 0<X_{i} \leq a_{i} x\right\} \\
& \quad=\operatorname{Pr}\left\{0<X_{i} \leq a_{i} x\right\} \cdot \operatorname{Pr}\left\{X_{j} \leq 0 \text { for } j \neq i \mid 0<X_{i} \leq a_{i} x\right\} .
\end{aligned}
$$

As $x \rightarrow 0$, we have

$$
\operatorname{Pr}\left\{0<X_{i} \leq a_{i} x\right\}=\frac{a_{i} x}{\sqrt{2 \pi \sigma_{i i}}}+O\left(x^{2}\right),
$$

and

$$
\operatorname{Pr}\left\{X_{j} \leq 0 \text { for } j \neq i \mid 0<X_{i} \leq a_{i} x\right\}=P_{i}+O(x),
$$

from Theorem 2 , which follows. Thus, the term (20) contributes $a_{i} P_{i} x / \sqrt{2 \pi \sigma_{i i}}+O\left(x^{2}\right)$ to the formula (19). This completes the proof.

Theorem 2: Define, for $h \geq 0$,

$$
P_{i}(h)=\operatorname{Pr}\left\{X_{j} \leq 0, \text { for } j \neq i \mid 0<X_{i} \leq h\right\} .
$$

Also, define the random variables $Y_{j}$, for $j \neq i$, by

$$
Y_{j}=\sigma_{i i} X_{j}-\sigma_{i j} X_{i} \text {. }
$$

Then,

$$
\lim _{h \rightarrow 0} P_{i}(h)=P_{i}=\operatorname{Pr}\left\{Y_{j} \leq 0, \text { for } j \neq i\right\}
$$

Also,

$$
\left|P_{i}(h)-P_{i}\right|=O(h) .
$$

Proof: If we can show that

$$
\operatorname{Pr}\left\{Y_{j} \leq-\left|\sigma_{i j}\right| h, \text { for } j \neq i\right\} \leq P_{i}(h) \leq \operatorname{Pr}\left\{Y_{j} \leq\left|\sigma_{i j}\right| h \text {, for } j \neq i\right\},
$$

then (21) follows immediately. We first note that if $0<X_{i} \leq h$, for each $j \neq i$, we have the following set inclusions:

$$
\left\{Y_{j} \leq-\left|\sigma_{i j}\right| h\right\} \subseteq\left\{X_{j} \leq 0\right\} \subseteq\left\{Y_{j} \leq\left|\sigma_{i j}\right| h\right\} .
$$

From this, it follows that

$\operatorname{Pr}\left\{Y_{j} \leq-\left|\sigma_{i j}\right| h\right.$, for $\left.j \neq i \mid 0<X_{i} \leq h\right\} \leq P_{i}(h)$

$$
\leq \operatorname{Pr}\left\{Y_{j} \leq\left|\sigma_{i j}\right| h, \text { for } j \neq i \mid 0<X_{i} \leq h\right\} .
$$

Now if $i \neq j, X_{i}$ and $Y_{j}$ are uncorrelated since

$$
E\left(X_{i} Y_{j}\right)=\sigma_{i i} E\left(X_{i} X_{j}\right)-\sigma_{i j} E\left(X_{i}^{2}\right)=\sigma_{i i} \sigma_{i j}-\sigma_{i j} \sigma_{i i}=0,
$$

so that the conditions in (24) can be deleted, giving (23). To prove the estimate (22), note that the set difference $\left\{Y_{j} \leq\left|\sigma_{i j}\right| h\right\}$ $-\left\{Y_{j} \leq-\left|\sigma_{i j}\right| h\right\}$ is contained in the union $\bigcup_{i=1}^{M-1}\left\{-\left|\sigma_{i j}\right| h \leq Y_{j}\right.$ $\left.\leq\left|\sigma_{i j}\right| h\right\}$, so that the difference between the right and left sides of (23) is bounded by

$$
\sum_{i=1}^{M-1} \operatorname{Pr}\left\{-\left|\sigma_{i j}\right| h \leq Y_{j} \leq\left|\sigma_{i j}\right| h\right\} .
$$

And finally, each term in (25) is $O(h)$. This proves (22).

\section{APPENDIX B}

\section{DERIVATIONS OF (9) AND (10)}

If the code $C$ has no zero columns and no repeated columns, then it is easily shown that

$$
\begin{aligned}
& \sum_{i=1}^{M-1} x_{i j}^{2}=2^{k-1}, \quad \text { for } j=1,2, \cdots, n, \\
& \sum_{i=1}^{M-1} x_{i j} \bar{x}_{i j}=0, \quad \text { for } j=1,2, \cdots, n,
\end{aligned}
$$

$$
\begin{aligned}
& \sum_{i=1}^{M-1} x_{i j} x_{i l}=2^{k-2}, \quad \text { for } j, l=1,2, \cdots, n \quad \text { and } j \neq l, \\
& \sum_{i=1}^{M-1} x_{i j} \bar{x}_{i l}=2^{k-2}, \quad \text { for } j, l=1,2, \cdots, n \text { and } j \neq l,
\end{aligned}
$$

where $x_{i j}, j=1,2, \cdots, n$, are the components of $x_{i}$ and $\bar{x}_{i j}$ is the complement of $x_{i j}$. Note that the symmetric assumption we made about each bit position of the codeword implies that there are no zero columns.

We have

$$
\sum_{i=1}^{M-1} d_{i}^{2}=\sum_{i=1}^{M-1}\left(\sum_{j=1}^{n} x_{i j}\right)\left(\sum_{m=1}^{n} x_{i m}\right) .
$$

If $j=m$, then by (26)

$$
\sum_{j=1}^{n} \sum_{i=1}^{M-1} x_{i j}^{2}=n 2^{k-1} .
$$


On the other hand, if $j \neq m$, then by (28)

$$
\sum_{j=1}^{n} \sum_{\substack{m=1 \\ m \neq j}}^{n} \sum_{i=1}^{M-1} x_{i j} x_{i m}=n(n-1) 2^{k-2} .
$$

Therefore,

$$
\begin{aligned}
\sum_{i=1}^{M-1} d_{i}^{2} & =n 2^{k-1}+n(n-1) 2^{k-2} \\
& =n(n+1) 2^{k-2} .
\end{aligned}
$$

This ends the derivation of (9).

Similarly,

$$
\sum_{i=1}^{M-1} d_{i} d\left(\boldsymbol{x}_{i} \oplus \boldsymbol{x}_{l}\right)=\sum_{i=1}^{M-1}\left(\sum_{j=1}^{n} x_{i j}\right)\left(\sum_{m=1}^{n}\left(x_{i m} \oplus x_{l m}\right)\right) .
$$

If $j=m$, then by (26) and (27)

$$
\sum_{i=1}^{M-1} x_{i j}\left(x_{i j} \oplus x_{l j}\right)=\left\{\begin{array}{l}
2^{k-1}, \quad \text { if } x_{l j}=0, \\
0, \quad \text { if } x_{l j}=1 .
\end{array}\right.
$$

Since there are $\left(n-d_{l}\right)$ of $x_{l j}$ 's such that $x_{l j}=0$,

$$
\sum_{j=1}^{n} \sum_{i=1}^{M-1} x_{i j}\left(x_{i j} \oplus x_{i j}\right)=\left(n-d_{l}\right) 2^{k-1} .
$$

On the other hand, if $j \neq m$, then by (28) and (29)

$$
\sum_{i=1}^{M-1} x_{i j}\left(x_{i m} \oplus x_{l m}\right)=2^{k-2} \text {. }
$$

It follows that

$$
\sum_{j=1}^{n} \sum_{\substack{m=1 \\ m \neq j}}^{n} \sum_{i=1}^{M-1} x_{i j}\left(x_{i m} \oplus x_{l m}\right)=n(n-1) 2^{k-2}
$$

Finally,

$$
\begin{aligned}
\sum_{i=1}^{M-1} d_{i} d\left(x_{i} \oplus x_{l}\right) & =\left(n-d_{l}\right) 2^{k-1}+n(n-1) 2^{k-2} \\
& =n(n+1) 2^{k-2}-d_{l} 2^{k-1},
\end{aligned}
$$

which is the result of (10).

\section{APPENDIX C}

\section{PRoOF OF THEOREM 5}

The Farkas Alternative [10, p. 56]: Either the equation

$$
\boldsymbol{A x}=\boldsymbol{b} \text { has a solution } \boldsymbol{x} \geq 0
$$

or (exclusively)

$$
\boldsymbol{y}^{T} \boldsymbol{A} \geq 0, \quad \boldsymbol{y}^{T} \boldsymbol{b}<0 \quad \text { has a solution } \boldsymbol{y} .
$$

Lemma 1: Either the equation

$$
A \boldsymbol{x}+\dot{\alpha} \boldsymbol{d}=\boldsymbol{b} \quad \text { has a solution } \boldsymbol{x} \geq 0, \quad \alpha \in R
$$

or (exclusively)

$$
\boldsymbol{y}^{T} \boldsymbol{A} \leq 0, \quad \boldsymbol{y}^{T} d=0, \quad \boldsymbol{y}^{T} \boldsymbol{b}>0 \quad \text { has a solution } \boldsymbol{y} .
$$

Proof: Set the unconstrained $\alpha=u-v$ in (30) and require $u \geq 0$ and $v \geq 0$. Now this lemma follows from the Farkas Alternative.

Theorem 5: Let the set $\mathscr{A}=\left\{x: A x \leq 0, d^{T} x=0\right\}$ be nonempty. The inequality $\boldsymbol{b}^{T} x \leq 0$ holds for all $x \in \mathscr{A}$, if and only if $b=A^{T} y+\alpha d$, for some $y \geq 0$ and $\alpha \in R$.

Proof: The proof for the sufficient condition is straightforward. Now suppose the necessary condition is wrong, and assume that $b$ is not in the form of $\boldsymbol{A}^{T} \boldsymbol{y}+\alpha \boldsymbol{d}, \boldsymbol{y} \geq 0$ and $\alpha \in R$. Then, the equation $\boldsymbol{A}^{T} \boldsymbol{y}+\alpha \boldsymbol{d}=\boldsymbol{b}$ does not have a solution $y \geq 0, \alpha \in R$. Therefore, the case (30) of Lemma 1 is wrong, and we must have the alternative:

$$
\boldsymbol{x}^{T} \boldsymbol{A}^{T} \leq 0, \quad \boldsymbol{x}^{T} \boldsymbol{d}=0, \quad \boldsymbol{x}^{T} \boldsymbol{b}>0 \text { has a solution } \boldsymbol{x},
$$

which contradicts the assumption that $\boldsymbol{b}^{T} x \leq 0$ holds for all $\boldsymbol{x} \in \mathscr{A}$.

\section{REFERENCES}

[1] E. C. Posner, "Properties of error-correcting codes at low signalto-noise ratios," SIAM J. Appl. Math., vol. 15, pp. 775-798, July 1967.

[2] W. Feller, An Introduction to Probability Theory and Its Applications, vol. 2, 2nd ed. New York: Wiley, 1971.

[3] F. J. MacWilliams and N. J. A. Sloane, The Theory of ErrorCorrecting Codes. Amsterdam: North Holland, 1977.

[4] J. H. van Lint, Introduction to Coding Theory. New York: Springer-Verlag, 1982.

[5] W. W. Peterson and E. J. Weldon, Jr., Error Correcting Codes, 2nd ed. Cambridge, MA: MIT Press, 1972.

[6] J. S. Leon, "Computing automorphism groups of error-correcting codes," IEEE Trans. Inform. Theory, vol. IT-28, pp. 496-511, May 1982.

[7] J. J. Costa, "An algorithm for finding the automorphism group of a linear code," Ph.D. dissert., Univ. of Southern California, Los Angeles, 1985.

[8] C.-C. Lu, "The automorphism groups of binary primitive $\mathrm{BCH}$ codes," Ph.D. dissert., Univ. of Southern California, Los Angeles, 1987.

[9] J. Franklin, Methods of Mathematical Economics. New York: Springer-Verlag, 1980.

[10] A. J. Viterbi and J. K. Omura, Principles of Digital Communication and Coding. New York: McGraw-Hill, 1979.

[11] A. J. Viterbi, "On coded phase-coherent communications," IRE Trans. Space Electron. Teleme., vol. SET-7, pp. 3-14, Mar. 1961 